\title{
Dexamethasone accelerates muscle regeneration by modulating kinesin-1-mediated focal adhesion signals
}

\author{
Jong-Wei Lin', Yi-Man Huang', Yin-Quan Chen ${ }^{2,3}$, Ting-Yun Chuang', Tien-Yun Lan', Yen-Wenn Liu', Hung-Wei Pan", \\ Li-Ru You', Yang-Kao Wang ${ }^{5}$, Keng-hui Lin', Arthur Chiou ${ }^{3}$ and Jean-Cheng Kuo (1) ${ }^{1,2}$
}

\begin{abstract}
During differentiation, skeletal muscle develops mature multinucleated muscle fibers, which could contract to exert force on a substrate. Muscle dysfunction occurs progressively in patients with muscular dystrophy, leading to a loss of the ability to walk and eventually to death. The synthetic glucocorticoid dexamethasone (Dex) has been used therapeutically to treat muscular dystrophy by an inhibition of inflammation, followed by slowing muscle degeneration and stabilizing muscle strength. Here, in mice with muscle injury, we found that Dex significantly promotes muscle regeneration via promoting kinesin-1 motor activity. Nevertheless, how Dex promotes myogenesis through kinesin-1 motors remains unclear. We found that Dex directly increases kinesin-1 motor activity, which is required for the expression of a myogenic marker (muscle myosin heavy chain 1/2), and also for the process of myoblast fusion and the formation of polarized myotubes. Upon differentiation, kinesin-1 mediates the recruitment of integrin $\beta 1$ onto microtubules allowing delivery of the protein into focal adhesions. Integrin $\beta 1$-mediated focal adhesion signaling then guides myoblast fusion towards a polarized morphology. By imposing geometric constrains via micropatterns, we have proved that cell adhesion is able to rescue the defects caused by kinesin-1 inhibition during the process of myogenesis. These discoveries reveal a mechanism by which Dex is able to promote myogenesis, and lead us towards approaches that are more efficient in improving skeletal muscle regeneration.
\end{abstract}

\section{Introduction}

Skeletal muscle fibers (muscle cells) develop when the myogenic program is activated resulting in a repression of cell proliferation and a stimulation of differentiation ${ }^{1-3}$. During muscle differentiation, several steps which change the structural and physiological functioning of the cytoskeleton are involved. These result in the evolution of single cells into multinucleated muscle fibers. In detail, myoblasts form cell adhesion organelles, namely focal adhesions (FAs), by engagement with the extracellular

\footnotetext{
Correspondence: Jean-Cheng Kuo (jckuo@nycu.edu.tw)

${ }^{1}$ Institute of Biochemistry and Molecular Biology, National Yang Ming Chiao

Tung University, Hsinchu 30010, Taiwan

${ }^{2}$ Cancer Progression Research Center, National Yang Ming Chiao Tung

University, Hsinchu 30010, Taiwan

Full list of author information is available at the end of the article

Edited by Maria Victoria Niklison Chirou
}

matrix (ECM); the later (FAs) enable the myoblasts to align with each other. After this, their actin filaments, which are anchored to the FAs, begin to form the myofibrils. During the next step, the differentiated myocytes fuse with each other to form multinucleated myotubes that contain strong myofibrils, with contractile capacity, essential for the muscle to function ${ }^{4-10}$. The main purpose of muscle fibers is to develop force by contracting relative to their surrounding ECM, such that the human body and other animals could support themselves and move.

The contractile apparatus in muscle fibers is driven by cytoskeleton structures ${ }^{11,12}$. Actin filaments interact with FAs to generate force and they serve as the major functional cytoskeletal component within a muscle fiber ${ }^{13-15}$. FAs start to form when integrin receptors engage with the

\section{(c) The Author(s) 2021}

(c) (i) Open Access This article is licensed under a Creative Commons Attribution 4.0 International License, which permits use, sharing, adaptation, distribution and reproduction cc) in any medium or format, as long as you give appropriate credit to the original author(s) and the source, provide a link to the Creative Commons license, and indicate if changes were made. The images or other third party material in this article are included in the article's Creative Commons license, unless indicated otherwise in a credit line to the material. If material is not included in the article's Creative Commons license and your intended use is not permitted by statutory regulation or exceeds the permitted use, you will need to obtain permission directly from the copyright holder. To view a copy of this license, visit http://creativecommons.org/licenses/by/4.0/. 
ECM and begin to be activated. Next, a series of FAassociated proteins are recruited to connect with the actin cytoskeleton $^{16-21}$. Under biochemical stimuli, as well as physical stimuli, the size and composition of FAs are regulated spatio-temporally in a process called FA maturation ${ }^{22-26}$. Modulation of the maturation state of the FAs plays a key determinant role in the biological responses downstream of integrin engagement, such as cell mobility ${ }^{27}$, stem cell differentiation lineage determination ${ }^{28}$, and myogenic differentiation ${ }^{29}$. The fact that integrin $\beta 1$ is required for myoblast fusion has been demonstrated both in vivo and in vitro ${ }^{30}$. In addition, these integrin-mediated signals, via focal adhesion kinase (FAK) activation, have been shown to play crucial roles in myoblast fusion in vitro and in muscle regeneration in vivo ${ }^{29}$. Therefore, FA organization is directly involved in the transmission of integrin-mediated signaling, which, in turn, plays a crucial role in controlling the process of muscle differentiation.

The microtubules present in muscle cells are extensively remodeled, and aligned parallel to the major cell axis; this orientation is central to determine the overall shape of a cell during myogenesis. Microtubule motor protein KIF5B, the expressed isoform of kinesin-1 heavy chain, which is abundant in skeletal muscle ${ }^{31}$, has been demonstrated to be responsible for muscle development $^{32}$. A study using mice with Kif5b conditionally knocked out in skeletal muscles shows that loss of kinesin-1 causes the aggregation of nuclei, mitochondria, and myofibril components, including $\alpha$-sarcomeric actin and non-muscle myosin IIB, within the cell body. This, in turn, results in impaired myofibrils assembly and muscle fiber terminal disturbances, leading to lateral detachment of myofibrils from the sarcolemma, with integrins still present on the sarcolemma ${ }^{32}$. This reveals the connection between integrins and actin filaments lost due to the loss of kinesin-1. It implies that the purpose of kinesin- 1 is to maintain the structural organization of the integrinmediated adhesion organelles FAs in skeletal muscles to retain the integration of myofibrils to integrins at sarcolemma. Other studies have indicated that, in normal skeletal muscle, integrin $\beta 1$ and various $F A$-associated proteins, including paxillin, talin, and vinculin, localize at the myotendinous junctions and form physical links between the sarcomeric units and the sarcolemma ${ }^{33}$. These findings support the idea that kinesin- 1 controls FA formation and the transmission of integrin-mediated FA signals during myogenesis. However, whether kinesin-1 regulates myogenesis through integrin-mediated FA signaling has never been investigated.

Muscle function loss happens in people with muscular dystrophy, the main clinical features of which are progressive loss of skeletal muscle and prominent muscle inflammation ${ }^{34-40}$; these eventually lead to the loss of the ability to walk and then death. To date, there is no treatment available to stop or to reverse any type of muscular dystrophy. Nevertheless, the synthetic glucocorticoid, such as dexamethasone (Dex), has been applied therapeutically to slow down muscle degeneration and stabilize muscle strength ${ }^{41,42}$. In addition, Dex seems to enhance the myogenic fusion efficiency of mouse myoblasts $\mathrm{C} 2 \mathrm{C} 12$ cells $^{43}$ via unknown mechanisms. Here, in the present study, we have demonstrated that Dex is sufficient to promote muscle regeneration in vivo and that this is mediated via kinesin-1 motor activity. We sought to investigate whether Dex regulates kinesin-1 motor activity, and further explored whether integrin $\beta 1$-mediated FA signaling is related to kinesin-1 in promoting myogenic differentiation.

\section{Results}

\section{Dexamethasone enhances kinesin-1 motor activity}

Previous studies have shown that Dex enhances myogenic differentiation ${ }^{43}$. To confirm this effect, $\mathrm{C} 2 \mathrm{C} 12$ cells were exposed to differentiation media containing the indicated concentrations of Dex for 0-5 days, and the expression of the myogenic marker myosin heavy chain $1 /$ 2 (MYH1/2) was accessed. Dex treatment induced expression of $\mathrm{MYH} 1 / 2$ during the early phase of the C2C12 differentiation time course (Supplemental Fig. 1a), indicating that Dex facilitated myogenic differentiation. On Day 5 of the time course, cells were stained to detect MYH1/2 and with DAPI to examine whether Dex promoted the fusion of mono-nuclear cells to form multinuclei myotubes (Supplemental Fig. 1b). By counting the number of nuclei per MYH1/2+ cell, we quantified myogenic fusion efficiency (fusion index). Higher myogenic fusion percentage was identified in the cells treated with Dex (Supplemental Fig. 1c). This confirmed that Dexinduced myogenic differentiation of $\mathrm{C} 2 \mathrm{C} 12$ cells.

Mitochondria activity is important for myogenic differentiation $^{44}$, and therefore we sought to determine the effect of Dex on the mitochondria function in the $\mathrm{C} 2 \mathrm{C} 12$ cells. We first examined whether Dex changed the abundance of mitochondria present during myogenic differentiation. To do this, we measured the ratio of cytochrome $\mathrm{C}$ oxidase I DNA (to indicate mitochondrial DNA; mtDNA) to $18 \mathrm{~S}$ rRNA DNA (to indicate nuclear DNA; nDNA) in $\mathrm{C} 2 \mathrm{C} 12$ cells that had been cultured in differentiation media with the indicated concentrations of Dex for 1 or 5 days. We found that Dex did not influence the abundance of mitochondria during myogenic differentiation (Fig. 1a). However, we detected higher ATP production in $\mathrm{C} 2 \mathrm{C} 12$ cells treated with Dex by measuring oxygen consumption rate (OCR) using Seahorse XF24 Extracellular Flux analysis (Fig. 1b). Mitochondria are highly dynamic organelles in the cells ${ }^{45}$. Evidence has indicated that changing mitochondrial motility often affects signaling pathways and cell functioning in many 

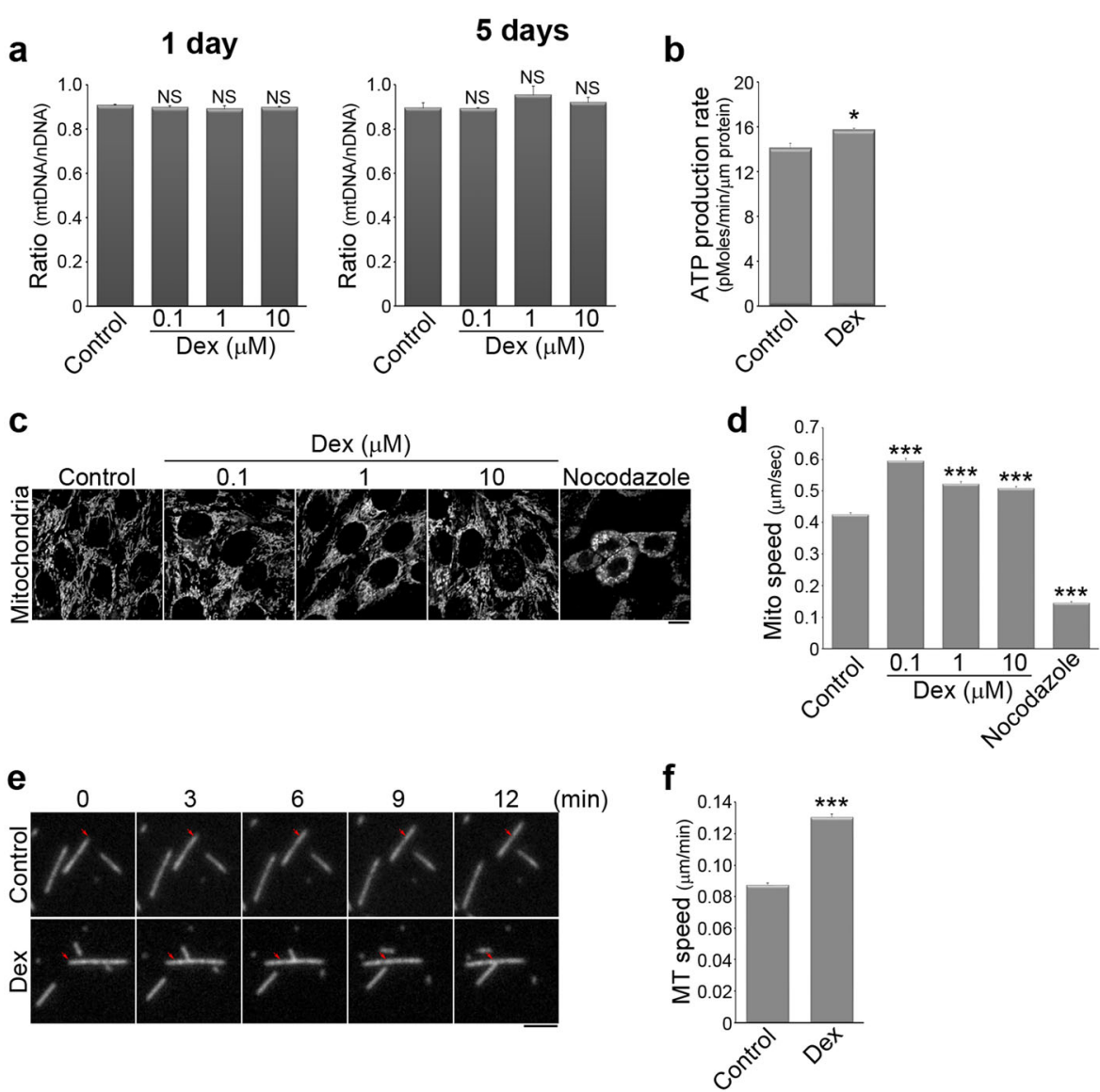

Fig. 1 Dexamethasone promotes mitochondria dynamics and enhances kinesin-1 motor activity. a C2C12 cells treated with DMSO (control), $0.1,1$, or $10 \mu \mathrm{M}$ Dex in differentiation medium for 1 day or 5 days had their mitochondrial numbers assessed using the ratio of cytochrome $C$ oxidase $I$ DNA (to indicate mitochondrial DNA; mtDNA) to $18 \mathrm{~S}$ rRNA DNA (to indicate nuclear DNA; nDNA) by real-time PCR. Data are mean \pm s.e.m ( $n=3$ independent experiments). NS no significance (compared with the control). $\mathbf{b}$ ATP production rate was calculated from oxygen consumption rate (OCR) in C2C12 cells treated with DMSO (control) or $1 \mu \mathrm{M}$ Dex for $6 \mathrm{~h}$. OCR was measured continuously followed by the addition of oligomycin $(1 \mu \mathrm{M})$, Carbonyl cyanide 4-(trifluoromethoxy) phenylhydrazone (FCCP; $1 \mu \mathrm{M})$, and antimycin A $(0.5 \mu \mathrm{M})$ with rotenone $(0.5 \mu \mathrm{M})$ using Seahorse XF 24 Extracellular Flux Analyzer. Data are mean \pm s.e.m ( $n=3$ independent experiments). ${ }^{*} p<0.05$ (compared with control). c C2C12 cells treated with DMSO (control), $0.1 \mu \mathrm{M}$ Dex, $1 \mu \mathrm{M}$ Dex, $10 \mu \mathrm{M}$ Dex for $1 \mathrm{~h}$, or with $10 \mu \mathrm{M}$ nocodazole for $16 \mathrm{~h}$ were stained with MitoTracker Red to visualize mitochondria. Scale bar, $10 \mu \mathrm{m}$. $\mathbf{d}$ Comparison of the average values for the speed of mitochondrial movement in C2C12 cells. Data are mean \pm s.e.m (control, $n=3638$ mitochondria/5 cells; $0.1 \mu \mathrm{M}$ Dex $n=5016$ mitochondria/6 cells; $1 \mu \mathrm{M}$ Dex, $n=3635$ mitochondria/ 6 cells; $10 \mu \mathrm{M}$ Dex, $n=4818$ mitochondria/ 6 cells; nocodazole, $n=632$ mitochondria/2 cells). ${ }^{* * *} p<0.001$ (compared with the control). e Time-lapse epi-fluorescence microscopy showing the movement of rhodamine-labeled microtubules treated with control (DMSO) or $1 \mu$ M Dex, using in vitro kinesin-1 motility assay. Scale bar, $2 \mu \mathrm{m}$. $\mathbf{f}$ Comparison of the average values for the speed of microtubule movement brought about by kinesin-1. Data are mean \pm s.e.m (control, $n=494$ microtubules; $1 \mu \mathrm{M}$ Dex, $n=370$ microtubules). ${ }^{* * *} p<0.001$ (compared with control).

different ways ${ }^{46}$. We next examined whether Dex affected mitochondrial dynamics in $\mathrm{C} 2 \mathrm{C} 12$ cells. We performed livecell imaging using MitoTracker Red to label the mitochondria of living cells (Fig. 1c). Using a time-lapse image series that showed the presence of MitoTracker Red in $\mathrm{C} 2 \mathrm{C} 12$ cells, we tracked mitochondrial motion using a previously developed software package, namely Mytoe ${ }^{47}$. The results allowed us to quantify the movement speed of individual mitochondria. Compared to the control cells, Dex-treated cells showed significantly higher mitochondrial speeds (Fig. 1d). Thus, Dex stimulates the mitochondria in C2C12 cells and increases their motility.
Microtubule motor kinesin-1 (also call KIF5B or conventional kinesin) is known to drive mitochondrial movement along microtubules ${ }^{48,49}$. We confirmed that depolymerization of microtubules by nocodazole significantly reduced the movement speed of the mitochondria (Fig. 1c, d). Therefore, we hypothesized that Dex affected kinesin-1 motors, which led to faster mitochondrial movement and myogenic differentiation. We therefore used an in vitro kinesin-1 motility assay with fluorescence-labeled microtubules (rhodamine-labeled purified tubulin) added onto the surface of coverslips containing coupled kinesin-1 motors. Using time-lapse 
epi-fluorescent image series of the rhodamine-labeled microtubules, the kinesin-1-induced motility of the microtubules was tracked (Fig. 1e). The results revealed that Dex significantly increased the speed of kinesin- 1 as it moved along microtubules. The increase was from $\sim 0.088 \mu \mathrm{m} / \mathrm{min}(0.088 \mu \mathrm{m} / \mathrm{min} \pm 0.001)$ to $\sim 0.131 \mu \mathrm{m} /$ $\min (0.131 \mu \mathrm{m} / \mathrm{min} \pm 0.002$; Fig. 1f). These results show that Dex increases kinesin-1 motor activity.

\section{Dexamethasone enhances muscle regeneration in vivo through kinesin-1}

We next sought to determine whether Dex activates kinesin-1 motors, and further affects skeletal muscle regeneration in vivo. We established a $\mathrm{BaCl}_{2}$-induced muscle injury model using 9-week-old C57BL/6 female mice. The mice with their tibialis anterior (TA) muscles in the left hind legs injured (by $1.2 \% \mathrm{BaCl}_{2}$ ) were treated with DMSO (the carrier control), Dex or Dex+ Rose Bengal Lactone (kinesin-1 inhibitor; RBL) everyday (Fig. 2a). The centrally nucleated myofibers, indicative of muscle regenerating were counted in $\mathrm{H} \& \mathrm{E}$ staining of TA muscle transverse sections (Fig. 2b). Compared with DMSO-treated mice, the Dex-treated mice showed a significantly larger number of centrally nucleated myofibers at 5 and 7 days post injury. At 10 days after injury, we observed that, in mice treated with Dex, most regenerating myofibers contained peripheral nuclei, indicating the role of Dex in promoting the formation and maturation of myofibers. In order to confirm that kinesin-1's motor activity mediates the effect of Dex during muscle regeneration, we used an kinesin-1 inhibitor, RBL, to disrupt the association between kinesin-1 and microtubules ${ }^{50,51}$. The combined treatment of Dex and RBL significantly reduced the number of centrally nucleated myofibers at 5 and 7 days post injury, compared with Dex-treated mice, which supports the hypothesis that disrupting kinesin-1 motor activity retards the improved skeletal muscle regeneration caused by Dex treatment (Fig. 2c). These findings confirm that Dex activates kinesin-1 in skeletal muscle to promote myofiber regeneration after muscle injury, and highlight the importance of exploring Dex-activated kinesin-1 in promoting myogenic differentiation.

\section{The acceleration of myotube differentiation by dexamethasone requires kinesin-1 sliding along microtubules}

To examine the direct effect of Dex on kinesin-1 motors in promoting myogenic differentiation, we generated nonsilenced and kinesin-1-silenced $\mathrm{C} 2 \mathrm{C} 12$ cells using a lentivirial short hairpin RNA (shRNA) (Fig. 3a). We first examined whether the observed rapid mitochondrial movement induced by Dex (Fig. 1c, d) was due to a direct up-regulation of kinesin-1 motor activity. These two types of cells were stained with MitoTracker Red in order to monitor the movement of individual mitochondria. We found that the increase in mitochondrial speed brought about by treatment with Dex was absent in the kinesin-1-silenced cells (Fig. 3b). When assessed using the mitochondrial stain Tom 20, we observed that treatment with Dex led to a dramatic redistribution of the mitochondria towards the cell periphery in non-silenced cells. Furthermore, depletion of kinesin-1 was able to suppress this effect of Dex, resulting in a marked accumulation of mitochondria close to the nucleus (Fig. 3c). By quantifying the area of mitochondria spreading as a percentage of each cell, we confirmed that Dex increased the area across which mitochondria were distributed in nonsilenced cells, but not in the kinesin-1-silenced cells (Fig. 3d). Specifically, the depletion of kinesin-1 substantially shrank the area of spread of the mitochondria, as shown in Fig. 3d. These results confirm that Dex affects mitochondrial movement by directly promoting kinesin- 1 motors activity.

Given that inhibition of microtubule depolymerization with nocodazole (Supplemental Fig. 2) and depletion of kinesin-1 (Fig. 3e) inhibited myogenic differentiation, we sought to determine the role of kinesin-1 in Dex-mediated myogenic differentiation. Non-silenced and kinesin-1silenced cells were exposed to differentiation media containing the indicated concentrations of Dex. Then, an analysis of MYH1/2 expression was carried out (Fig. 3e) in parallel with measurement of the myogenic fusion indices of the various treatment groups (Fig. 3f, g). We found that silencing of kinesin-1 significantly suppressed both MYH1/2 expression (Fig. 3e) and myocyte fusion in $\mathrm{C} 2 \mathrm{C} 12$ cells that had been treated with Dex (Fig. 3f, g), which reveals the essential role that kinesin-1 plays in Dex-mediated myogenic differentiation. Subsequently, C2C12 cells were exposed for 3 days to differentiation media containing the indicated concentrations of Dex and kinesin-1 inhibitor RBL ${ }^{50,51}$. We found that RBL dramatically inhibited Dex-induced MYH1/ 2 expression in a dose-dependent manner (Fig. 3h). After being placed in the differentiation medium with $40 \mu \mathrm{M}$ RBL for 3 days, protein down-regulation of kinesin- 1 and $\alpha$ tubulins was detected (Fig. 3i). We also measured the cells' myogenic fusion indices, and confirmed that disruption of kinesin-1 moving along the microtubules caused a dramatic reduction in Dex-induced multi-nuclei myotubes formation (Fig. 3j, k). These results support the hypothesis that Dex affects myogenic differentiation via direct activation of kinesin-1 motors.

\section{Kinesin-1 is responsible for integrin $\beta 1$ transportation, which acts as a focal adhesion signal}

Kinesin-1 has been implicated in generating and stabilizing muscle cell organization in mice ${ }^{32}$. In kinesin-1silenced $\mathrm{C} 2 \mathrm{C} 12$ cells cultured for 5 days in differentiation media, we detected fewer cells that expressed the myogenic marker MYH1/2 compared to non-silenced cells 

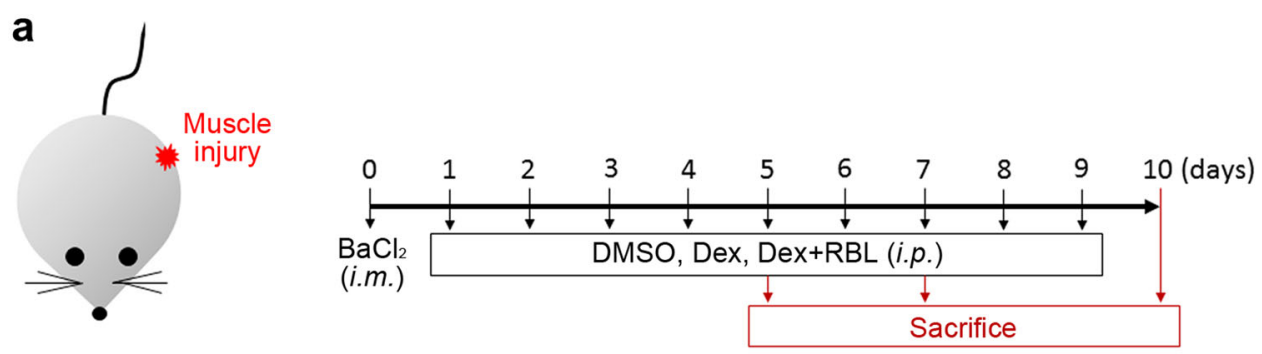

b $\quad$ DMSO

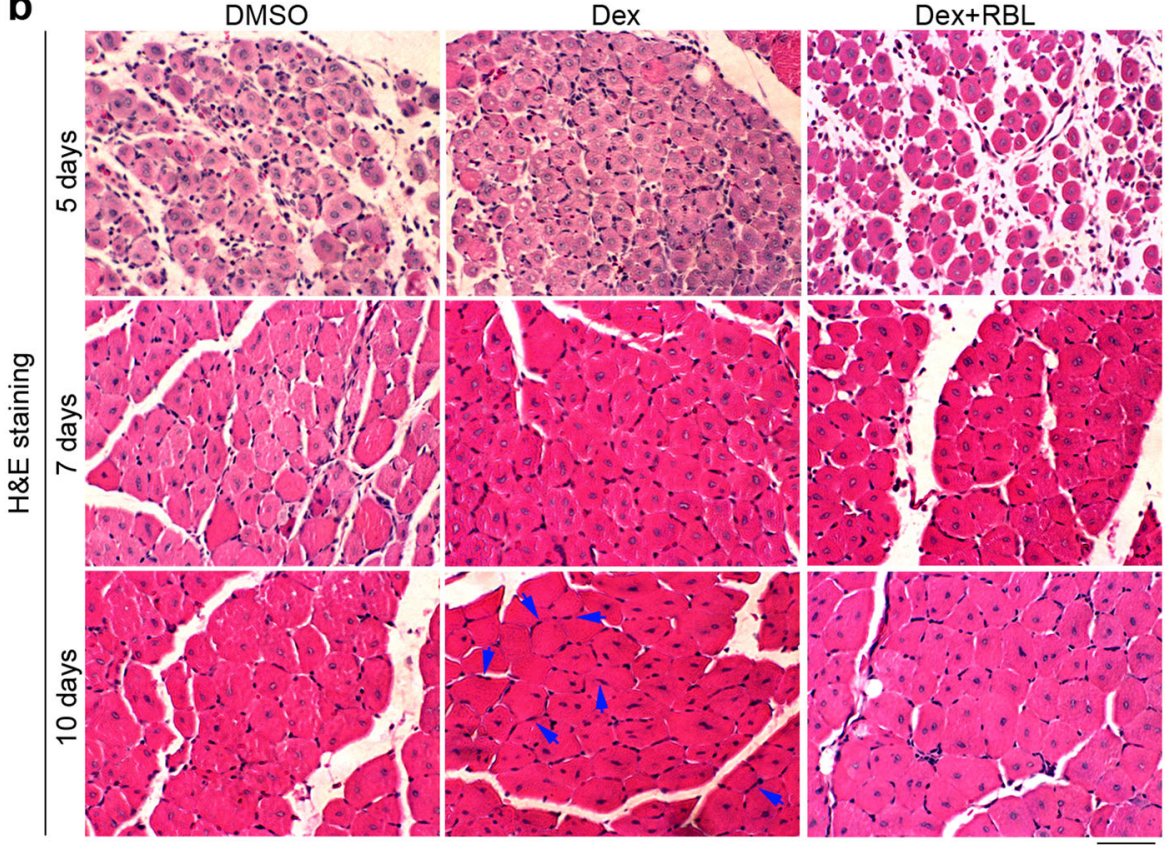

C

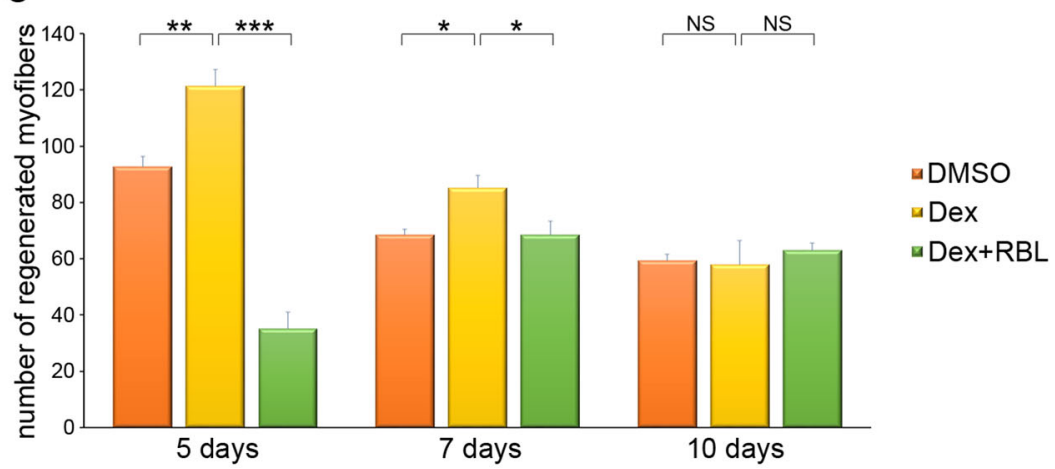

Fig. 2 Kinesin-1 drives muscle regeneration in mice. a Experimental scheme for the skeletal muscle regeneration experiments. The tibialis anterior (TA) muscles in the left hind legs of mice were injured using $1.2 \% \mathrm{BaCl}_{2}$ via intramuscular (i.m.) injection on day 0 . Next, the mice were randomly grouped and administered with DMSO, Dex $(0.1 \mathrm{mg} / \mathrm{kg})$ or Dex $(0.1 \mathrm{mg} / \mathrm{kg})+\mathrm{RBL}(30 \mathrm{mg} / \mathrm{kg})$ everyday via intraperitoneal (i.p.) injection. To assess whether Dex is able to accelerate skeletal muscle regeneration via kinesin-1, mouse TA muscle samples were collected at various time points.

b Representative histology images of mouse TA muscles of the various study groups. The regenerating myofibers with peripheral nuclei marked with blue arrows. Scale bar, $50 \mu \mathrm{m}$. c The number of regenerative muscle fibers, calculated as the number of fibers with accumulation of centrally located nuclei, as shown in b. Data are mean \pm s.e.m $\left(n=5\right.$ independent fields). ${ }^{*} p<0.05 ;{ }^{* *} p<0.01 ;{ }^{* * *} p<0.001$; NS no significance.

(Fig. 3f). Of these kinesin-1-silenced $\mathrm{C} 2 \mathrm{C} 12$ cells showing $\mathrm{MYH} 1 / 2^{+}$expression, we quantified their aspect ratio, and found that their aspect ratio was lower than that of non-silenced $\mathrm{C} 2 \mathrm{C} 12$ cells; this was true for cells both with less than three nuclei and those with more than three nuclei (Fig. 4a). This points to the fact that the elongation 


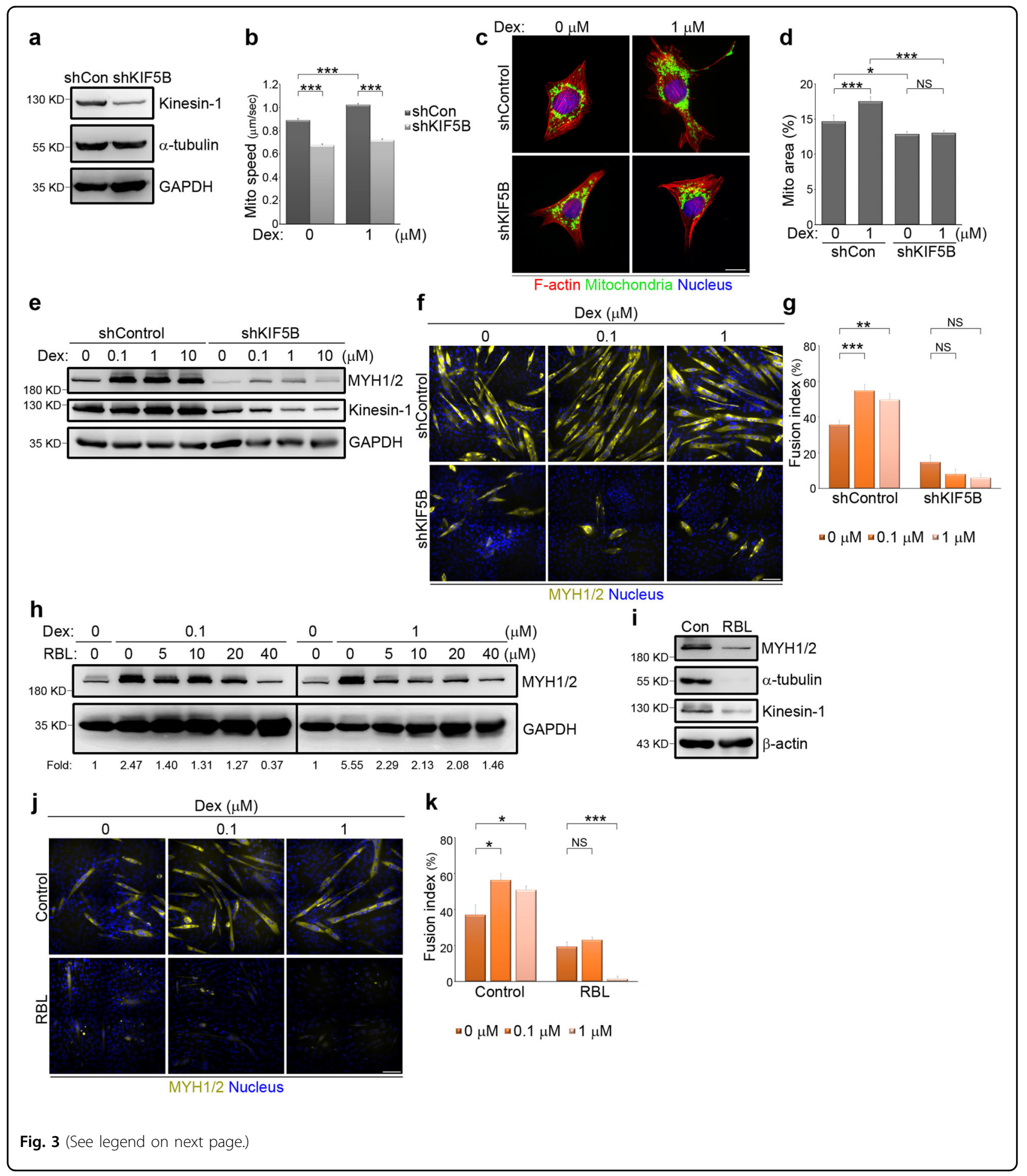

of cells, which is related to fusion, was blocked in these kinesin-1-silenced cells, which in turn supports a previous observation that kinesin-1-deficient mouse muscle fibers display shrunken ends and lateral detachment from the sarcolemma ${ }^{32}$. Based on the above, we next focused on
FAs; this was because these integrin-based adhesive structures are known to act as a key regulator of integrin activation, and it is quite possible that FAs are regulated by kinesin-1. We acquired TIRF images of differentiated $\mathrm{C} 2 \mathrm{C} 12$ cells to show the FA marker paxillin and active 
(see figure on previous page)

Fig. 3 Kinesin- 1 controls dexamethasone-promoted myogenic differentiation. a Total cell lysate from C2C12 cells expressing non-silenced (shCon) or Kinesin-1-silenced (shKIF5B) shRNAs were analyzed by western blotting using antibodies against kinesin-1, a-tubulin, and GAPDH. b Effects of kinesin-1-silencing on Dex-enhanced mitochondria dynamics. C2C12 cells expressing non-silenced (shCon) or Kinesin-1-silenced (shKIF5B) shRNAs were treated with the indicated concentrations of Dex for $1 \mathrm{~h}$. Data are mean \pm s.e.m (shCon in $0 \mu \mathrm{M}$ Dex, $n=3481$ mitochondria/6 cells; shCon in $1 \mu \mathrm{M}$ Dex, $n=4716$ mitochondria/6 cells; shKIF5B in $0 \mu \mathrm{M}$ Dex, $n=3847$ mitochondria/ 6 cells; shKIF5B in $1 \mu \mathrm{M}$ Dex, $n=3578$ mitochondria/ 6 cells). ${ }^{* * *} p<0.001$. c C2C12 cells expressing non-silenced (shControl) or Kinesin-1-silenced (shKIF5B) shRNAs were treated with the indicated concentrations of Dex for $6 \mathrm{~h}$ and immunostained for Tom20 (green; to visualize mitochondria), phalloidin (red; to visualize F-actin), and DAPI (blue; to visualize nucleus). Scale bar, $10 \mu \mathrm{m}$. d The mitochondria area (marked with Tom20) as a percentage within a cell (marked with phalloidin), as shown in c. Data are mean \pm s.e.m (shCon in $0 \mu \mathrm{M}$ Dex, $n=29$ cells; shCon in $1 \mu \mathrm{M}$ Dex, $n=30$ cells; shKIF5B in $0 \mu \mathrm{M}$ Dex, $n=30$ cells; shKIF5B in $1 \mu \mathrm{M}$ Dex, $n=30$ cells). ${ }^{*} p<0.05 ;{ }^{* * *} p<0.001$; NS no significance. e Effects of kinesin-1 silencing on Dex-enhanced myogenic differentiation. C2C12 cells expressing non-silenced (shControl) or Kinesin-1-silenced (shKIF5B) shRNAs were treated with differentiation medium containing the indicated concentrations of Dex for 3 days and analyzed by western blotting using antibodies against MYH1/2, kinesin-1, and GAPDH. $\mathbf{f}$ Effects of kinesin-1 on Dex-promoted myoblast fusion. C2C12 cells expressing non-silenced (shControl) or Kinesin-1-silenced (shKIF5B) shRNAs were treated with differentiation medium containing the indicated concentrations of Dex for 5 days and immunostained for MYH1/2 (yellow; myotube marker) and DAPI (to visualize nucleus). Scale bar, $100 \mu \mathrm{m}$. g Fusion index, calculated as the percentage of nuclei $(\geq 3)$ in $\mathrm{MYH} 1 / 2^{+}$cells, as shown in $\mathbf{f}$. Data are mean \pm s.e.m (shControl in $0 \mu \mathrm{M}$ Dex, $n=313 \mathrm{MYH} 1 / 2^{+}$cells; shControl in $0.1 \mu \mathrm{M}$ Dex, $n=236 \mathrm{MYH1} / 2^{+}$cells; shControl in $1 \mu \mathrm{M}$ Dex, $n=192 \mathrm{MYH1} / 2^{+}$cells; shKIF5B in $0 \mu \mathrm{M}$ Dex, $n=104 \mathrm{MYH1} / 2^{+}$cells; shKIF5B in $0.1 \mu \mathrm{M}$ Dex, $n=84 \mathrm{MYH1} / 2^{+}$cells; and shKIF5B in $1 \mu \mathrm{M} \mathrm{Dex}, n=79 \mathrm{MYH1} / 2^{+}$cells). ${ }^{* *} p<0.01 ;{ }^{* * *} p<0.001$; NS no significance. $\mathbf{h}$ Effects of the association between kinesin-1 and microtubules on Dex-enhanced myogenic differentiation. C2C12 cells were treated with differentiation medium containing the indicated concentrations of Dex and kinesin-1 inhibitor RBL for 3 days and analyzed by western blotting using antibodies against MYH1/2, and GAPDH (internal control). The ratio of MYH1/2 to GAPDH is shown as fold. $\mathbf{i}$ C2C12 cells were treated with differentiation medium containing $40 \mu \mathrm{M}$ RBL for 3 days and analyzed by western blotting using antibodies against MYH1/2, a-tubulin, kinesin-1, and $\beta$-actin. $\mathbf{j}$ Effects of the association between kinesin-1 and microtubules on Dex-promoted myoblast fusion. C2C12 cells were treated with differentiation medium containing the indicated concentrations of Dex accompanied with either DMSO (control) or $40 \mu \mathrm{M}$ RBL for 5 days and immunostained for MYH1/2 (yellow; myotube marker) and DAPI (to visualize nucleus). Scale bar, $100 \mu \mathrm{m}$. $\mathbf{k}$ Fusion index, calculated as the percentage of nuclei $(\geq 3)$ in MYH1/2+ cells, as shown in $\mathbf{j}$. Data are mean \pm s.e.m (DMSO in $0 \mu \mathrm{M}$ Dex, $n=144 \mathrm{MYH1} / 2^{+}$cells; DMSO in $0.1 \mu \mathrm{M}$ Dex, $n=160$ $\mathrm{MYH} 1 / 2^{+}$cells; DMSO in $1 \mu \mathrm{M}$ Dex, $n=157 \mathrm{MYH1} / 2^{+}$cells; RBL in $0 \mu \mathrm{M}$ Dex, $n=130 \mathrm{MYH} 1 / 2^{+}$cells; RBL in $0.1 \mu \mathrm{M}$ Dex, $n=164 \mathrm{MYH} 1 / 2^{+}$cells; RBL in $1 \mu \mathrm{M}$ Dex, $n=34 \mathrm{MYH1} / 2^{+}$cells). ${ }^{*} p<0.05 ;{ }^{* * *} p<0.001$; NS no significance.

integrin $\beta 1$ (9EG7). In non-silenced cells, we observed strong clustering of active integrin $\beta 1$ and paxillin to form fibrillar FAs, particularly at the edge of the myotubes, and these FAs connected to bundled actin filaments. In contrast, in the kinesin-1-silenced cells, there was very limited clustering of active integrin $\beta 1$ and paxillin, and as a result there were only a few FAs present; furthermore, only weak actin filaments were formed (Fig. 4b). These results reveal that kinesin-1 expression controls both the activation and clustering of integrin $\beta 1$, and thereby allowing FA formation in the differentiated $\mathrm{C} 2 \mathrm{C} 12$ cells. A similar effect was observed when kinesin-1 motor activity was abolished by RBL treatment (Fig. 4c). We next sought to determine whether kinesin-1 plays a crucial role in regulating integrin $\beta 1$-mediated signaling within FAs by measuring the level of FAK phosphorylation at tyrosine $397^{52,53}$. We found that both silencing of kinesin-1 and disruption of its motor activity by RBL, independently, led to a significant decrease in phosphorylation levels of FAK at tyrosine 397 in the differentiated $\mathrm{C} 2 \mathrm{C} 12$ cells (Fig. 4d, e). These results support the hypothesis that kinesin-1, via its motor activity, regulates the transmission of the integrin $\beta 1$ mediated signals that bring about FA assembly and maturation, and that this in turn controls the elongation of cells during the process of myogenic differentiation.

Considering the critical role of integrin $\beta 1$ clustering in promoting FAK phosphorylation at tyrosine $397^{52,53}$, we next investigated whether disruption of kinesin-1, and the resulting myogenic defects, could be restored by activating integrin $\beta 1$. C2C12 cells were transfected with wildtype integrin $\beta 1$ (integrin $\beta 1 \mathrm{wt}$ ), auto-clustering integrin $\beta 1$ (integrin $\beta 1 \mathrm{~V} 737 \mathrm{~N}$ ), or constitutively active integrin $\beta 1$ (integrin $\beta 1 \mathrm{G} 429 \mathrm{~N}$ ). We observed that RBL treatment inhibited MYH1/2 expression in integrin $\beta 1$ wtexpressing cells after 3 days in differentiation media. However, expression of either integrin $\beta 1$ V737N or integrin $\beta 1 \mathrm{G} 429 \mathrm{~N}$ in $\mathrm{C} 2 \mathrm{C} 12$ cells failed to rescue the RBL-induced myogenic defect (Fig. 4f). Dissociating kinesin-1 and microtubules by the use of RBL has been shown to cause protein down-regulation of kinesin-1 and $\alpha$-tubulin (Fig. 3i). We also found protein downregulation of exogenous integrin $\beta 1$ wt, exogenous integrin $\beta 1 \mathrm{~V} 737 \mathrm{~N}$, and exogenous integrin $\beta 1 \mathrm{G} 429 \mathrm{~N}$ (Fig. 4f). These results suggest that, during myogenic differentiation, integrin $\beta 1$ transport along microtubules may happen in association with kinesin-1. Indeed, upon myogenic induction, an increased level of integrin $\beta 1$ in the polymerized microtubule-containing fraction (pellet; insoluble fraction) was detected using a previously published microtubule isolation method ${ }^{54}$ (Fig. 4g). However, this effect was abolished in the kinesin-1-silenced cells (Fig. 4g). These findings confirm that integrin $\beta 1$ is transported along the microtubules through kinesin-1 during the process of myogenic differentiation.

The above results show that, in the presence of RBL, either exogenous auto-clustering integrin $\beta 1$ or 


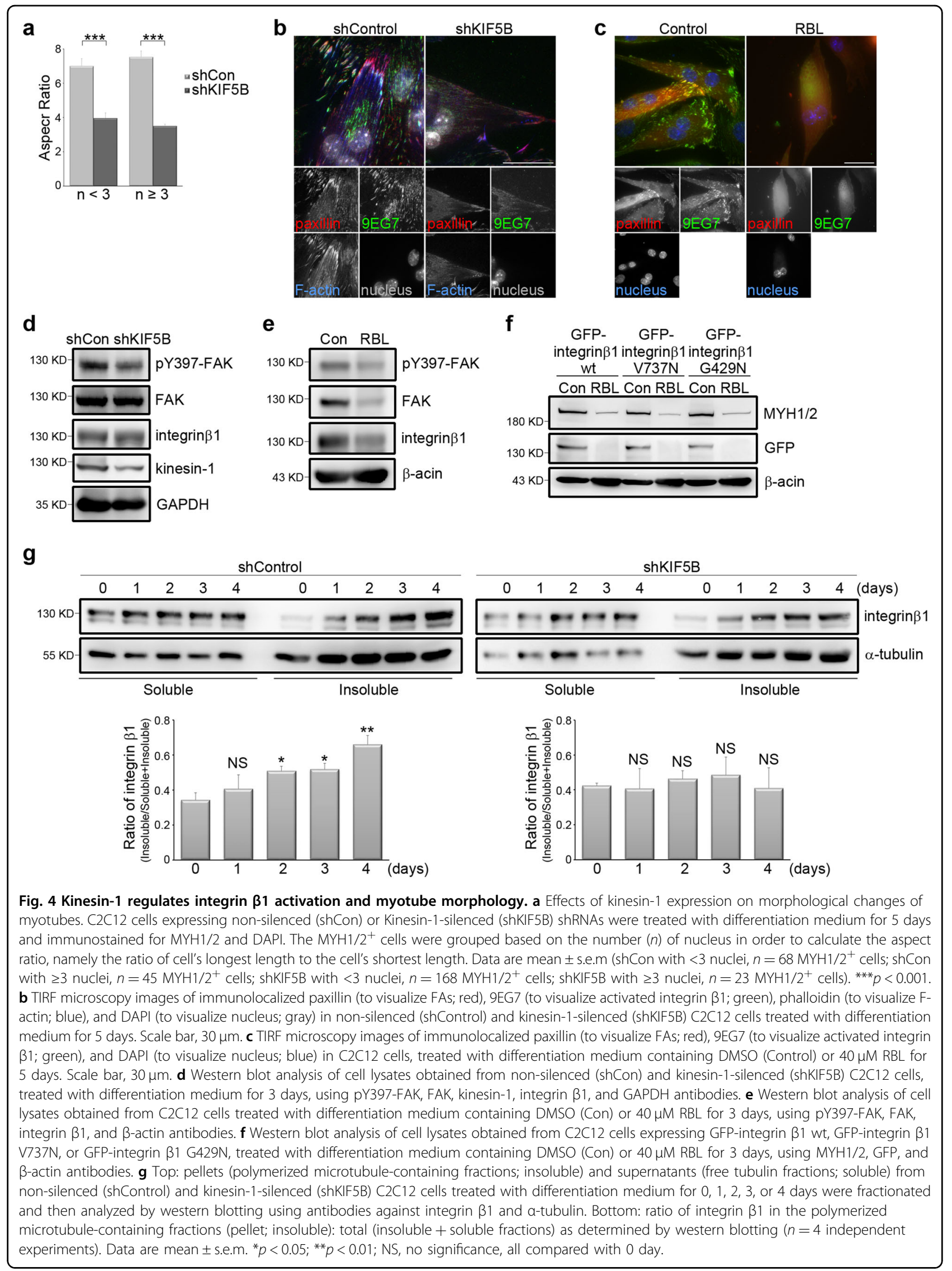


constitutively active integrin $\beta 1$ is down-regulated and is not able to restore myogenic defects (Fig. 4f). We then hypothesized that kinesin-1 mediates integrin $\beta 1$ transport to transmit the FA-related signals that not only shape cells into an elongated morphology, but also deliver the signals needed for myogenic differentiation. In order to examine further whether kinesin-1 disruption causes myogenic defects via a reduction in cell adhesion (which is integrin $\beta 1$ - dependent), we used microcontact printing techniques (see Materials and methods section) to generate stripes of fibronectin on polydimethylsiloxane (PDMS) substrate (Fig. 5a, b); the width of these stripes was set to either $50 \mu \mathrm{m}$ or $20 \mu \mathrm{m}$. Non-silenced and kinesin-1-silenced cells were then plated on fibronectincoated coverslips (with no pattern substrate), $20 \mu \mathrm{m}$ striped coverslips or $50 \mu \mathrm{m}$ striped coverslips; these cells were then cultured in differentiation media. After 5 days, cells were stained with MYH1/2 protein and nuclei (Fig. 5c). We then quantified the aspect ratio of the identified $\mathrm{MYH} 1 / 2^{+}$cells, and found that both $20 \mu \mathrm{m}$ and $50 \mu \mathrm{m}$ fibronectin-coated stripes were able to shape the kinesin1-silenced cells into an elongated morphology, including both cells with fewer than three nuclei and cells with three or more nuclei (Fig. 5d). An assessment of the distribution of mitochondria in the above three types of cells, using Tom 20 staining (Fig. 5e), revealed that the reduction in spreading area of the mitochondria caused by kinesin-1 depletion was rescued when the kinesin-1-silenced cells were plated on either $20 \mu \mathrm{m}$ or $50 \mu \mathrm{m}$ fibronectin-coated stripes (Fig. 5f). Next, we measured the myogenic fusion indices of the various types of cells and the results confirmed that the $20 \mu \mathrm{m}$ and $50 \mu \mathrm{m}$ fibronectin-coated stripes significantly restored the defect in multi-nuclei myotube formation found in kinesin-1-silenced cells (Fig. $5 \mathrm{~g})$. Therefore, it is clear that the fibronectin-coated stripes were able to elongate the cells, and that cell adhesion-mediated cell polarity can restore the defects (caused by kinesin-1 depletion), including mitochondrial distribution and myogenic differentiation.

\section{Discussion}

The goal of this study was to clarify the mechanism by which Dex regulates myogenic differentiation. To achieve this goal, we first used an in vitro kinesin-1 activity assay to confirm the direct effect of Dex on kinesin-1 motors activation. Upon Dex stimulation, the speed of kinesin-1 moving along the microtubules was accelerated, which also increased mitochondrial dispersion (Fig. 6a). In mice with muscle injury, we observed that Dex treatment promoted the regeneration of skeletal muscles via kinesin1 motor activity (Fig. 2). Therefore, Dex is likely to regulate muscle differentiation by activating kinesin-1. These observations highlight the importance of exploring how kinesin-1 activated by Dex affects myogenic differentiation. Indeed, we confirmed that, during myogenic differentiation, kinesin-1 was responsible for the transport of integrin $\beta 1$ along the microtubules to the plasma membrane, which in turn increased clustering of integrin $\beta 1$ on plasma membrane. The activation and clustering of integrin $\beta 1$ resulted in FA assembly, FA maturation, and the transmission of integrin $\beta 1$-mediated FA signals that in turn facilitated myogenic differentiation (Fig. 6b). These findings indicate, for the first time, that Dex is able to directly activate kinesin-1 motor proteins, thereby enhancing myogenic differentiation via FA signaling mediated by integrin $\beta 1$. Clarifying the important role of kinesin-1 in Dex-mediated myogenic differentiation has the potential to become an important therapeutic strategy when clinically managing muscle regeneration.

Previous studies have demonstrated that various glucocorticoids, including Dex, are able to affect mitochondrial energy production via both mitochondrial respiration and gluconeogenesis ${ }^{55}$. These metabolic states are modified by mitochondrial morphological adaptation, especially by dynamic events such as transport, fusion, fission, and quality control. The morphological change of mitochondria serves as a regulator in the process of myogenic differentiation ${ }^{44}$. In our study, we examined the positive effect of Dex and its beneficial role in myogenic differentiation (Supplemental Fig. 1a). We also found that mitochondrial transport was accelerated by Dex, and, in particular, that its transport speed was microtubule-dependent (Fig. 1c, d). Mitochondria are linked to the microtubule motor protein kinesin- $1^{48}$ via the adapter protein Milton and the mitochondrial membrane GTPase miro ${ }^{56,57}$, and thereby providing the possibility that Dex is able to act directly on the kinesin-1 motors. The assertion of this hypothesis was confirmed by in vitro kinesin-1 motility assays. By measuring the movement speed of rhodamine-labeled microtubules, we confirmed that Dex directly promoted kinesin-1 motor activity and accelerates the kinesin- 1 movement along the microtubules (Fig. 1e, f). Next, we used kinesin-1silenced $\mathrm{C} 2 \mathrm{C} 12$ cells, and found that Dex treatment resulted in blocking of the increased mitochondrial movement (Fig. 3b), the mitochondrial dispersion (Fig. 3c, d), and the myogenic differentiation (Fig. 3e-g). These findings support the notion that kinesin-1 is required for mitochondrial transport $^{48}$ and myogenic differentiation ${ }^{32}$. Altogether, we conclude that Dex is able to promote myogenic differentiation via a direct enhancement of kinesin-1 motility.

Integrin $\beta 1$-mediated FA signaling regulates myogenic differentiation ${ }^{29,30}$. Several studies have revealed that the major cell-ECM adhesive molecules in muscle are integrin $\beta 1$ with its different $\alpha$ subunits ${ }^{58}$; these are known to be required for myoblast fusion and sarcomere assembly ${ }^{30}$. Mice with an integrin $\beta 1$ deficiency in skeletal muscle die at birth with non-inflated lungs because of their inherent muscle defects, which supports the importance of integrin 


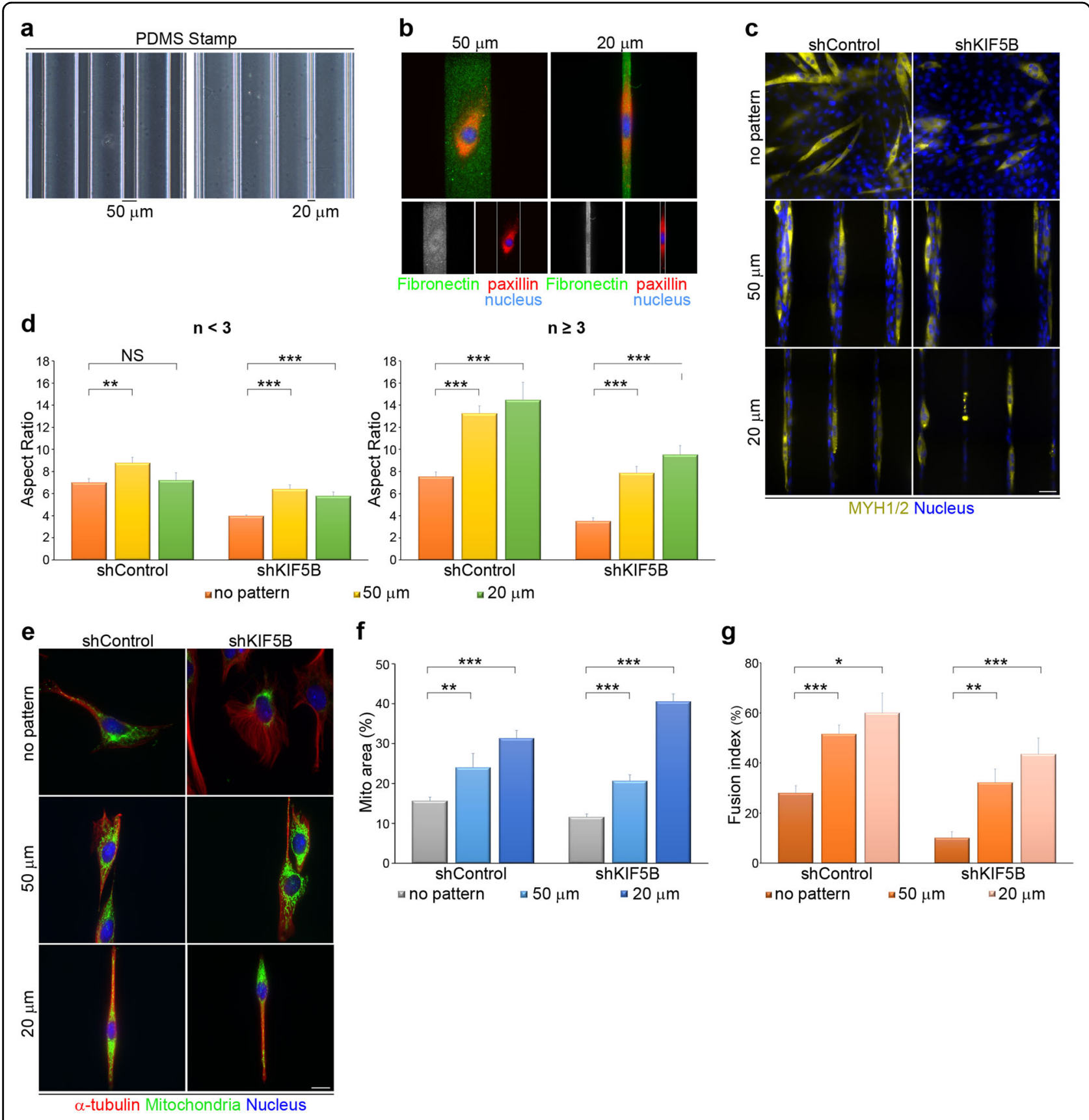

Fig. 5 (See legend on next page.)

$\beta 1$ in muscle development ${ }^{30}$. Integrin $\beta 1$-deficient muscle fibers lack a typical striate pattern and also show defects in the lateral linkage of the muscle fiber cytoskeleton to the sarcolemma ${ }^{30}$; these defects are similar to those found in kinesin-1-deficient skeletal muscle ${ }^{48}$. Despite the fact that the effects of integrin $\beta 1$ and kinesin- 1 in muscle development have been investigated previously, our study has revealed, for the first time, a novel mechanism whereby kinesin- 1 is able to regulate integrin $\beta 1$ activation at FAs (Fig. 4b, c) and for FA signals transmission (Fig. 4d, e). We have confirmed that integrin $\beta 1$ is transported along microtubules via kinesin-1 motors during myogenic differentiation (Fig. 4g), which explains why auto-clustering integrin $\beta 1$ (integrin $\beta 1 \mathrm{~V} 737 \mathrm{~N}$ ) and constitutively active integrin $\beta 1$ (integrin $\beta 1$ G429N) are unable to restore the myogenic defects caused by $\mathrm{RBL}$ 
(see figure on previous page)

Fig. 5 Geometric cue-coordinated cell adhesions are able to rescue the myogenic defects caused by kinesin-1 inhibition. a

A polydimethylsiloxane (PDMS) stamp with micron-sized features (20 $\mu \mathrm{m}$ or $50 \mu \mathrm{m}$ stripes) were shown in phase images. b Images of C2C12 cells plated on $20 \mu \mathrm{m}$ or $50 \mu \mathrm{m}$ stripe micropatterns; these were stained for fibronectin (to visualize stripe micropatterns; green), paxillin (to visualize FAs; red) and DAPI (to visualize nucleus; blue). c C2C12 cells expressing non-silenced (shControl) or kinesin-1-silenced (shKIF5B) shRNAs were plated on fibronectin-coated coverslips (with no pattern substrate), $20 \mu \mathrm{m}$ striped coverslips or $50 \mu \mathrm{m}$ striped coverslips, treated with differentiation medium for 5 days and immunostained for MYH1/2 (yellow; myotube marker) and DAPI (to visualize nucleus). Scale bar, $50 \mu$ m. $\mathbf{d}$ Aspect ratio, calculated as a ratio of the longest cell length to the shortest cell length in $\mathrm{MYH1} / 2^{+}$cells with nuclei $<3(n<3)$ or nuclei $\geq 3(n \geq 3)$, as shown in $\mathbf{c}$. Data are mean \pm s.e.m (shControl with no pattern with $<3$ nuclei, $n=68 \mathrm{MYH1} / 2^{+}$cells; shControl on $50 \mu \mathrm{m}$ stripes with $<3$ nuclei, $n=34 \mathrm{MYH} 1 / 2^{+}$cells; shControl on $20 \mu \mathrm{m}$ stripes with $<3$ nuclei, $n=24 \mathrm{MYH1} / 2^{+}$cells; shKIF5B with no pattern with $<3$ nuclei, $n=168 \mathrm{MYH1} / 2^{+}$cells; shKIF5B on $50 \mu \mathrm{m}$ stripes with $<3$ nuclei, $n=65 \mathrm{MYH1} / 2^{+}$cells; shKIF5B on $20 \mu \mathrm{m}$ stripes with $<3$ nuclei, $n=38 \mathrm{MYH1} / 2^{+}$cells; shControl with no pattern with $\geq 3$ nuclei, $n=45$ $\mathrm{MYH} 1 / 2^{+}$cells; shControl on $50 \mu \mathrm{m}$ stripes with $\geq 3$ nuclei, $n=52 \mathrm{MYH1} / 2^{+}$cells; shControl on $20 \mu \mathrm{m}$ stripes with $\geq 3$ nuclei, $n=21 \mathrm{MYH1/2^{+ }}$ cells; shKIF5B with no pattern with $\geq 3$ nuclei, $n=23 \mathrm{MYH1} / 2^{+}$cells; shKIF5B on $50 \mu \mathrm{m}$ stripes with $\geq 3$ nuclei, $n=25 \mathrm{MYH} 1 / 2^{+}$cells; shKIF5B on $20 \mu \mathrm{m}$ stripes with $\geq 3$ nuclei, $n=23 \mathrm{MYH1} / 2^{+}$cells). ${ }^{* *} p<0.01$; ${ }^{* * *} p<0.001$; NS no significance. e C2C12 cells expressing non-silenced (shControl) or kinesin-1-silenced (shKIF5B) shRNAs were plated on fibronectin-coated coverslips (with no pattern substrate), $20 \mu \mathrm{m}$ striped coverslips or $50 \mu \mathrm{m}$ striped coverslips for $24 \mathrm{~h}$ and immunostained for Tom20 (green; to visualize mitochondria), a-tubulin (red; to visualize microtubules), and DAPI (to visualize nucleus). Scale bar, $50 \mu \mathrm{m}$. f Percentage of mitochondria area (marked with Tom20) within a cell (marked with a-tubulin), as shown in e. Data are mean \pm s.e.m (shControl with no pattern, $n=32$ cells; shControl on $50 \mu \mathrm{m}$ stripes, $n=22$ cells; shControl on $20 \mu \mathrm{m}$ stripes, $n=20$ cells; shKIF5B with no pattern, $n=39$ cells; shKIF5B on $50 \mu \mathrm{m}$ stripes, $n=34$ cells; shKIF5B on $20 \mu \mathrm{m}$ stripes, $n=20$ cells). ${ }^{* *} p<0.01$; ${ }^{* * *} p<0.001$. g Fusion index, calculated as the percentage of nuclei $(\geq 3)$ in $\mathrm{MYH1} / 2^{+}$cells, as shown in c. Data are mean \pm s.e.m (shControl with no pattern, $n=154 \mathrm{MYH1} / 2^{+}$ cells; shControl on $50 \mu \mathrm{m}$ stripes, $n=112 \mathrm{MYH1} / 2^{+}$cells; shControl on $20 \mu \mathrm{m}$ stripes, $n=53 \mathrm{MYH1} / 2^{+}$cells; shKIF5B with no pattern, $n=165 \mathrm{MYH} 1 /$ $2^{+}$cells; shKIF5B on $50 \mu \mathrm{m}$ stripes, $n=108 \mathrm{MYH1} / 2^{+}$cells; shKIF5B on $20 \mu \mathrm{m}$ stripes, $n=56 \mathrm{MYH1} / 2^{+}$cells). ${ }^{*} p<0.05 ;{ }^{* *} p<0.01 ;{ }^{* * *} p<0.001$.

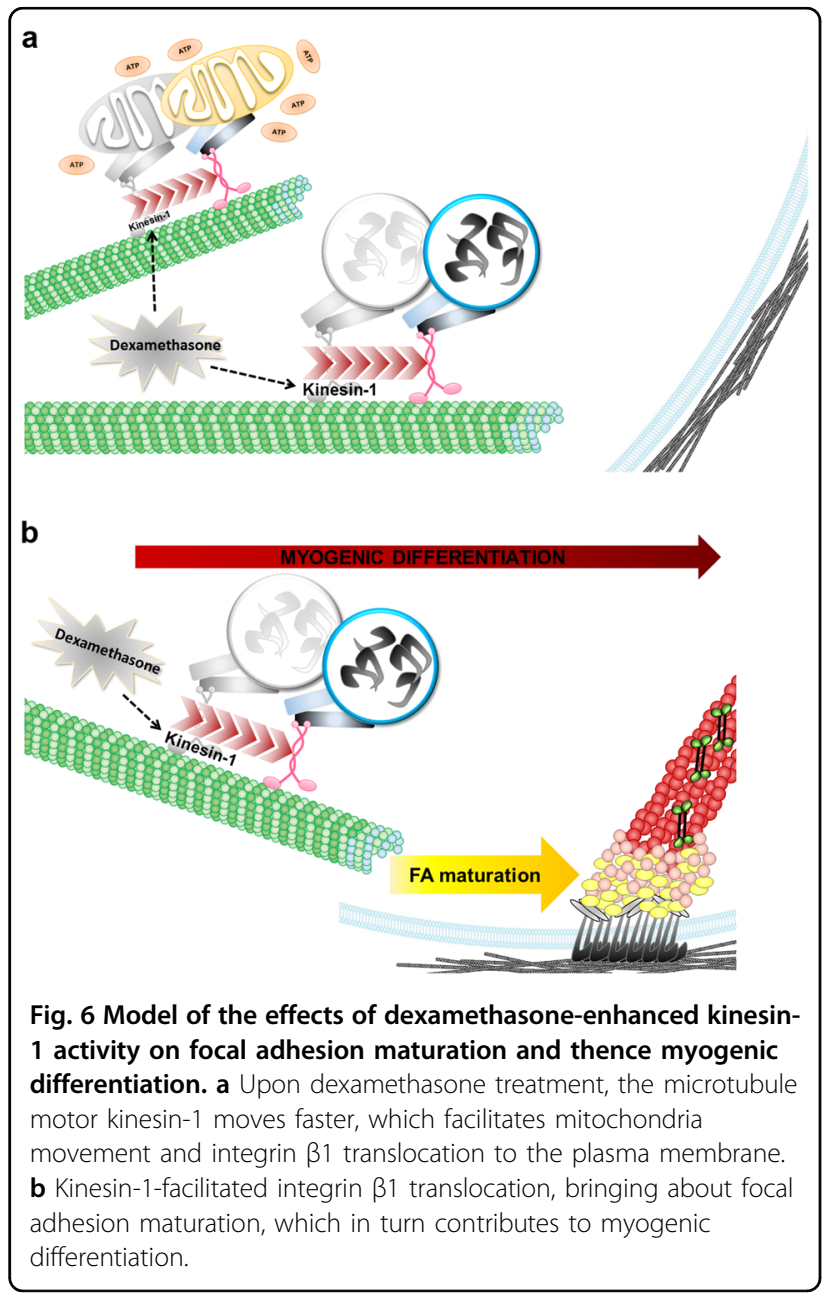

treatment (Fig. 4f). By plating $\mathrm{C} 2 \mathrm{C} 12$ cells onto fibronectin-coated microstripes, and thereby forcing integrin $\beta 1$ activation in cells with polarized morphology, the defects caused by kinesin-1 disruption in mitochondria distribution (Fig. 5e, f) and myogenic differentiation (Fig. 5g) can be rescued. This new finding indicates that integrin $\beta 1$ activation plays a crucial role in driving the myogenic machinery, and that this is regulated by microtubule motors kinesin- 1 .

The binding of kinesin-1 motor proteins to the microtubules is crucial to kinesin-1-driven transport, which is able to haul various molecular cargoes to specific reaction sites in cells. The binding influences microtubule stability. When kinesin- 1 shows strong binding to microtubules, it inhibits the shrinkage of microtubules and enhances the stability of their microtubule tracks. In the absence of kinesin binding, microtubules rapidly depolymerized ${ }^{59}$. We have shown that treatment with RBL inhibits kinesin1 activity by blocking the binding between kinesin- 1 and microtubules ${ }^{50}$, which, in turn, suppresses the protein levels of kinesin- 1 and $\alpha$-tubulin in $\mathrm{C} 2 \mathrm{C} 12$ cells (Fig. 3i). A previous study has revealed an enhancement of ubiquitinated c-MYC, p53, kinesin-1, and $\alpha$-tubulin in RBLtreated HEK293T cells ${ }^{51}$; thus, we assume that, in RBLtreated $\mathrm{C} 2 \mathrm{C} 12$ cells, kinesin- 1 and $\alpha$-tubulin are degraded via the proteasome pathway. Interestingly, we have also shown that the protein level of GFP-integrin $\beta 1 \mathrm{wt}$, GFPintegrin $\beta 1 \mathrm{~V} 737 \mathrm{~N}$ (the auto-clustering integrin $\beta 1$ ), and GFP-integrin $\beta 1$ G429N (constitutively active integrin $\beta 1$ ) decreases in the cells treated with RBL (Fig. 4f). Since we have confirmed the effect of kinesin- 1 on integrin $\beta 1$ transport along microtubules for FAs assembly and FA 
signals transmission, the dissociation of kinesin-1 from microtubules by RBL may cause integrin $\beta 1$ to deviate from its normal path and undergo proteasomal degradation. Therefore, further studies are needed to determine how kinesin-1 mediates integrin $\beta 1$ transport and how kinesin- 1 activity controls the proteasomal degradation of integrin $\beta 1$.

\section{Materials and methods}

\section{Cell culture}

The mouse myoblasts $\mathrm{C} 2 \mathrm{C} 12$ were kindly provided by Dr. Pei-Ching Chang (National Yang Ming Chiao Tung University, Taiwan). Cells have been tested and confirmed to be free of mycoplasma contamination. Cells were cultured in DMEM-high glucose (ThermoFisher) supplemented with 10\% FBS (ThermoFisher) and 1\% penicillin/ streptomycin (ThermoFisher). C2C12 differentiation was carried out using myogenesis induction medium (DMEMhigh glucose supplemented with $2 \%$ horse serum and $1 \%$ penicillin/streptomycin). C2C12 cells stably expressing non-silenced and KIF5B shRNA were generated using a lentiviral shRNA system according to the manufacturer's instructions (National RNAi Core Facility Platform/Academia Sinica). Transient transfections were performed using Lipofectamine 2000 (ThermoFisher). For all experiments, the cells were seeded on fibronectin-coated coverslips, plates, or microstripes.

\section{Plasmids and reagents Plasmids}

Expression silencing of kinesin-1 proteins was achieved using pLKO-KIF5B shRNA (TRCN0000332767, National RNAi Core Facility Platform). pLKO vector (TRCN000208001, National RNAi Core Facility Platform) was used as a control (non-silenced shRNA). The GFP-integrin $\beta 1 \mathrm{wt}$, GFP-integrin $\beta 1$ V737N, and GFP-integrin $\beta 1$ G429N were kindly provided by Dr. Ming-Jer Tang (National Cheng Kung University, Taiwan).

\section{Antibodies}

Mouse anti-paxillin (BD 610052); rat anti-active integrin $\beta 1$ (9EG7; BD 553715); rabbit-anti-Tom20 (Santa Cruz sc-11415); mouse-anti-MYH1/2 (Santa Cruz sc-53088); rabbit anti-fibronectin (Santa Cruz sc-9068); rabbit antiGAPDH (GeneTex GTX100118); rabbit anti- $\beta$-actin (GeneTex GTX100313); rabbit anti-integrin $\beta 1$ (GeneTex GTX128839); rabbit anti-paxillin (GeneTex GTX125891); rabbit anti-pY397-FAK (GeneTex GTX129840); rabbit anti-kinesin-1 (Abcam AB167429); rabbit anti-GFP (Abcam AB290); mouse anti- $\alpha$-tubulin (Sigma T5168); mouse anti-MYH2 (Thermo Fisher 14-6503-80); rabbit anti-FAK (Thermo Fisher AHO0502); Alexa Fluor 488 phalloidin (Thermo Fisher A12379); Alexa Fluor 568 phalloidin (Thermo Fisher A12380); Alexa Fluor 488-anti-rabbit
IgG (Thermo Fisher A11034); Alexa Fluor 488-anti-mouse IgG (Thermo Fisher A11029); Alexa Fluor 488-anti-rat IgG (Thermo Fisher A11006); Alexa Fluor 568-anti-rabbit IgG (Thermo Fisher A11036); Alexa Fluor 568-anti-mouse IgG (Thermo Fisher A11031); DAPI (Thermo Fisher D1306); Mitotracker Red (Thermo Fisher M7512); HRPAffiniPure mouse anti-rabbit IgG (Jackson ImmunoResearch 211-032-171); and HRP-AffiniPure goat anti-mouse IgG (Jackson ImmunoResearch 115-035-174).

\section{Reagents}

Nocodazole (Sigma); Dexamethasone (Dex; Sigma); Rose Bengal Lactone (RBL; Sigma); and Barium Chloride $\left(\mathrm{BaCl}_{2}\right.$; Sigma).

\section{RNA extraction, reverse transcription, and real-time quantitative PCR}

RNA was extracted from cells using TRIzol reagent (Invitrogen) and total RNA precipitated according to the manufacturer's instructions. The RNA products were reverse-transcribed using RevertAid First Strand cDNA Synthesis Kits (ThermoFisher) and random hexamer primers. The cDNA products were amplified by $\mathrm{PCR}$ using a KAPA SYBR® FAST qPCR Kits (ABI Prism; Roche). The 18SrRNA gene and cytochrome C oxidase I gene were used as indicators of nuclear DNA and mitochondrial DNA, respectively. Quantification of the target mRNA was carried out by the $\triangle \triangle C T$ method. The qPCR primers for the nuclear 18S rRNA gene were $5^{\prime}-\mathrm{T}$ AGAGGGACAAGTGGCGTTC- $3^{\prime}$ and 5'-CGCTGAG CCAGTCAGTGT- $3^{\prime}$. The qPCR primers for the mitochondrial cytochrome $\mathrm{C}$ oxidase I gene were $5^{\prime}$-GC CCCAGATATAGCATTCCC- $3^{\prime}$ and 5'-GTTCATCCTG TTCCTGCTCC-3'.

\section{Measurement of ATP production rate}

ATP production rate was calculated from oxygen consumption rate (OCR) using a Seahorse $\mathrm{XF}^{\mathrm{e}} 24$ Extracellular Flux Analyzer (Seahorse Bioscience) according to the manufacturer's protocol. $\mathrm{C} 2 \mathrm{C} 12$ cells were seeded in culture medium $24 \mathrm{~h}$ before measurement at a density of $3 \times 10^{4}$ cells per well, and then treated with DMSO or $1 \mu \mathrm{M}$ Dex for $6 \mathrm{~h}$ for analysis. Oligomycin $(1 \mu \mathrm{M})$, Carbonyl cyanide 4-(trifluoromethoxy)phenylhydrazone (FCCP; $1 \mu \mathrm{M})$, and antimycin A $(0.5 \mu \mathrm{M})$ with rotenone $(0.5 \mu \mathrm{M})$ were added sequentially to evaluate mitochondrial respiration. The data were normalized by the amount of protein present in each well.

\section{Kinesin-1 motility assay}

The protocol for kinesin-1 motility assay was followed according to manufacturer's instructions (BK027; Cytoskeleton). To prepare tubulin stock proteins, $60 \mu \mathrm{g}$ rhodamine tubulin (TL331M; Cytoskeleton) in $12 \mu \mathrm{l}$ ice-cold 
General Tubulin Buffer (BST01-001; Cytoskeleton) were mixed with $250 \mu \mathrm{g}$ unlabeled tubulin (TL238-A; Cytoskeleton) in $50 \mu \mathrm{l}$ ice-cold General Tubulin Buffer, and further mixed with $12 \mu \mathrm{l}$ ice-cold Microtubule Cushion Buffer (BK027-CB; Cytoskeleton). Immediately, the mixed proteins were split into $2 \mu \mathrm{l}$ aliquot, placed in individual eppendorf tube and frozen in liquid nitrogen for storage at $-70^{\circ} \mathrm{C}$. For the preparation of polymerized microtubule, $2 \mu \mathrm{l}$ of tubulin stock proteins were mixed with $2 \mu \mathrm{l}$ ice-cold Microtubule Cushion Buffer for $15 \mathrm{~min}$ at $35^{\circ} \mathrm{C}$. Subsequently, $100 \mu \mathrm{l}$ Taxol supplemented General Tubulin Buffer $(20 \mu \mathrm{M}$ Taxol in General Tubulin Buffer $)$ were added, and gently mixed. This step results in a population of Taxol-stabilized microtubules with an average length of 5 to $10 \mu \mathrm{m}$ and at a concentration of $7 \times$ $10^{10} / \mathrm{ml}$. To remove the un-polymerized tubulin to decrease the background fluorescence, $104 \mu \mathrm{l}$ of microtubule solution were carefully layered onto $400 \mu \mathrm{l}$ Taxol supplemented Microtubule Cushion Buffer $(20 \mu \mathrm{M}$ Taxol in Microtubule Cushion Buffer) into an ultracentrifugation tube (Beckman), and centrifuged at $100,000 \times g$ at $25^{\circ} \mathrm{C}$ for $30 \mathrm{~min}$ to pellet the microtubules. The microtubule pellets were gently re-suspended in $100 \mu \mathrm{l}$ Taxol supplemented General Tubulin Buffer. The Taxolstabilized microtubule stock population remains stable at room temperature for several hours. To perform the kinesin-1 motility assay, the kinesin-1 stock proteins were prepared by mixing $25 \mu \mathrm{g}$ recombinant kinesin- 1 motor domain proteins (KR01; Cytoskeleton) in $6 \mu \mathrm{l}$ Kinesin Buffer (BK027-KB; cytoskeleton), which was then split into $1 \mu \mathrm{l}$ aliquot in individual eppendorf tubes and then frozen in liquid nitrogen for storage at $-70^{\circ} \mathrm{C}$. One tube of kinesin-1 stock proteins was mixed with $11 \mu \mathrm{l}$ General Tubulin Buffer, which were perfused in the acid washed perfusion chamber (BSM05-04; Cytoskeleton), and incubated at room temperature for $5 \mathrm{~min}$. This was followed by blocking of the perfusion chamber with $11 \mu$ l blocking solution (BK027-BL; Cytoskeleton) at room temperature for $5 \mathrm{~min}$; $10 \mu \mathrm{l}$ fluorescent Taxol-stabilized microtubule stock was then perfused into the perfusion chamber and incubate for $5 \mathrm{~min}$ at room temperature. Subsequently, $20 \mu \mathrm{l}$ Wash buffer solution [17.8 $\mu$ l Chamber Wash Buffer (BK027-WB; Cytoskeleton), $0.2 \mu \mathrm{l} 2 \mathrm{mM}$ taxol and $2 \mu \mathrm{l}$ $10 \mathrm{x}$ antifade] was perfused into the chamber to remove any microtubules that were not bound to the kinesin-1 motor proteins. To activate the motility of the kinesin-1 motor proteins, $10 \mu \mathrm{l}$ motility buffer $(4.2 \mu \mathrm{l}$ General Tubulin Buffer, $4.2 \mu \mathrm{l}$ Chamber Wash Buffer, $0.1 \mu \mathrm{l} 2 \mathrm{mM}$ taxol, $1 \mu \mathrm{l} 10 \mathrm{X}$ antifade, and $0.5 \mu \mathrm{l} 100 \mathrm{mM}$ ATP) supplemented with DMSO or $1 \mu \mathrm{M}$ Dex was perfused into the chamber. The time-lapse epi-fluorescence images were obtained using a microscope (DMRBE, Leica) coupled with a 100XNA1.3 PL FLUOTAR objective lens (Leica) and an 512B EMCCD (Andor); these were operated by Micro-Manager 1.4 software (Leica). The time-lapse images showing microtubule movement were analyzed using Metamorph in order to assess the kinesin1 motor activity.

\section{Microtubule isolation}

$\mathrm{C} 2 \mathrm{C} 12$ cells were plated on culture dishes coated with $10 \mu \mathrm{g} / \mathrm{ml}$ fibronectin for $24 \mathrm{~h}$ at $50 \%$ confluence. The cells were then washed twice with $\mathrm{PBS}$ at $37^{\circ} \mathrm{C}$ and incubated with microtubule-stabilizing buffer (100 mM PIPES, $\mathrm{pH}$ 6.9, $5 \mathrm{mM} \mathrm{MgCl}_{2}$, 2 mM EGTA, $2 \mathrm{M}$ glycerol, 0.1\% NP40, $10 \mathrm{mM}$ beta-glycerophosphate, $50 \mathrm{mM} \mathrm{NaF}, 0.3 \mu \mathrm{M}$ okadaic acid, and $1 \mathrm{mM}$ PMSF) containing protease inhibitors and phosphatase inhibitors (Roche) for $15 \mathrm{~min}$ at $37^{\circ} \mathrm{C}$. Cell lysate was collected by scraping with a rubber policeman, and the suspension centrifuged at room temperature for $5 \mathrm{~min}$ at $1000 \times g$. After centrifugation, the supernatants (soluble fraction) were collected, and the pellets (insoluble fraction) were solubilized and sonicated in microtubule-stabilizing buffer for $15 \mathrm{~s}$ on ice.

\section{Time-lapse tracking to assess mitochondrial dynamics and related image analysis}

To analyze the dynamics of mitochondria, cells were stained with $25 \mathrm{nM}$ Mitotracker Red and mounted on a magnetic chamber (LCI) cultured in phenol red-free culture medium with $25 \mathrm{mM}$ Hepes $(\mathrm{pH}=7.4)$. Timelapse confocal images of Mitotracker Red were captured at $2 \mathrm{~s}$ intervals using an iLas multi-modal of TIRF (Roper)/spinning disk confocal (CSUX1, Yokogawa) microscope (Ti-E, Nikon) system equipped with $100 \times$ 1.49NA Plan objective lens (Nikon) and an EMCCD (ProEM, Princeton). To track and analyze the dynamics of Mitotracker Red, a mitochondria-tracking program Mytoe $^{47}$ was used to quantified mitochondria movement (mitochondria speed). The results are presented graphically using Excel software (Microsoft).

\section{Immunofluorescence analysis and image analysis}

For paxillin/active integrin $\beta 1$ (9EG7) staining, the cells were fixed with $4 \%$ paraformaldehyde in cytoskeleton buffer (10 mM MES pH 6.1, $138 \mathrm{mM} \mathrm{KCl,} 3 \mathrm{mM} \mathrm{MgCl}$, and $2 \mathrm{mM}$ EGTA) at room temperature for $20 \mathrm{~min}$, permeabilized with cytoskeleton buffer containing $0.5 \%$ Triton X-100 at room temperature for $5 \mathrm{~min}$, removed aldehyde groups with $0.1 \mathrm{M}$ glycine in PBS at room temperature for $10 \mathrm{~min}$, and blocked with blocking solution (3\% BSA/0.02\% Triton-X100 in PBS) at room temperature for $60 \mathrm{~min}$. Subsequently, the cells were incubated with the indicated primary antibodies in blocking solution at $4{ }^{\circ} \mathrm{C}$ for $16 \mathrm{~h}$, and then incubated with fluorescent dye-conjugated secondary antibody at room temperature for $1 \mathrm{~h}$. For mitochondria (Tom20) and MYH staining, the cells were fixed with $4 \%$ paraformaldehyde at 
room temperature for $20 \mathrm{~min}$, permeabilized with PBS containing $0.1 \%$ saponin at room temperature for $5 \mathrm{~min}$, removed aldehyde groups with $0.1 \mathrm{M}$ glycine in PBS at room temperature for $10 \mathrm{~min}$, and blocked with blocking solution (2\% BSA in PBS) at room temperature for $1 \mathrm{~h}$. Subsequently, the cells were incubated with the indicated primary antibodies in blocking solution at $4{ }^{\circ} \mathrm{C}$ for $16 \mathrm{~h}$, and then incubated with fluorescent dye-conjugated secondary antibody at room temperature for $1 \mathrm{~h}$. Last, the cells were mounted on coverslips using fluorescent mounting medium (DAKO), or on a magnetic chamber (LCI) and incubated with PBS containing $N$-propyl gallate for epi-fluorescence or TIRF imaging, respectively.

TIRF images were obtained using an iLas multi-modal of TIRF (Roper)/spinning disk confocal (CSUX1, Yokogawa) microscope (Ti-E, Nikon) system equipped with $60 \times 1.49$ NA or $100 \times 1.49$ NA Plan objective lens (Nikon) on an Evolve EMCCD (Photometrics) with an $\sim 100 \mathrm{~nm}$ evanescent field depth. TIRF images were captured and processed using Metamorph software. Epi-fluorescence images were obtained using a microscope (DMRBE, Leica) coupled with a $40 \times$ NA1.0 or $63 \times$ NA1.4 objective lens (Leica) and an 512B EMCCD (Andor); these were operated by Micro-Manager 1.4 software (Leica). Slidesscanned epi-fluorescence images were obtained using an epi-fluorescence microscope system (Ti-E, Nikon) coupled with a 40XNA1.3 or 100XNA1.49 Plan objective lens (Nikon) and a sCMOS camera (OHCA-Flash 4.0, 1024× 1024 pixels, Hamamatsu); these were operated by NISElements software (Nikon). H\&E staining of the samples of tibialis anterior (TA) muscles were obtained using an epi-fluorescence microscope system (TS100-F, Nikon) coupled with a 40XNA0.6 objective lens (Nikon) and a Whited WS-500 CCD camera.

\section{Fabrication of the micropatterned substrates}

The protocol for the fabrication of micropatterned substrates on PDMS was carried out as described previously $^{60}$. Briefly, PDMS stamps (linear fabricated stamps) were cast, baked, and removed from their silicon wafers (master templates), which was fabricated by photolithographic methods. The surface of the PDMS stamps were coated with fibronectin $(50 \mu \mathrm{g} / \mathrm{ml})$ for $1 \mathrm{~h}$, washed with sterilized $\mathrm{ddH}_{2} \mathrm{O}$, and dried with compressed air. A glass coverslip spin-coated with PDMS was UV oxidized for 10 min (UVO cleaner 42; Jelight), and then placed in contact with a PDMS stamps for $2 \mathrm{~min}$ in order to transfer the fibronectin. The coverslips were then blocked with $0.2 \%$ Pluronic ${ }^{\circledR}$ F-127 (Sigma) for $1 \mathrm{~h}$, and rinsed three times with PBS before cell seeding.

\section{Analysis of muscle regeneration}

For regeneration studies, tibialis anterior muscles in the left hind legs of 9-week-old C57BL/6 females mice were injured by intramuscular injection of $60 \mu \mathrm{l} 1.2 \% \mathrm{BaCl}_{2}$. All mice were randomly divided into three groups with five mice per group. Following the intraperitoneal injection of DMSO, Dex $(0.1 \mathrm{mg} / \mathrm{kg})$ or Dex $(0.1 \mathrm{mg} / \mathrm{kg})+\mathrm{RBL}$ $(30 \mathrm{mg} / \mathrm{kg})$ were administered everyday. The muscles were collected at the indicated time points, and embedded in paraffin for microtom sectioning. Regeneration after injury was analyzed in the hematoxylin and eosin (H\&E)stained muscle sections by measuring the number of centrally nucleated regenerating myofibers within the injured area. The centrally nucleated regenerating myofibers in 20X magnification field. For each sample, five fields were randomly selected and counted. The animal care and experimental protocols were approved by the Institutional Animal Care and Use Committee (IACUC) of National Yang Ming Chiao Tung University. The mice were housed on a 12-h light and 12-h dark cycle.

\section{Statistical analysis and data presentation}

Statistical significance was calculated by either the Student's $t$ test or one-way ANOVA. All the graphs were plotted using Excel software (Microsoft).

\section{Acknowledgements \\ J.C.K. is supported by research grants from the Taiwan Ministry of Science and Technology (109-2326-B-010 -002 -MY3), from the Veterans General Hospitals and University System of Taiwan Joint Research Program (VGHUST110-G7-6-1), from the Cancer Progression Research Center (National Yang Ming Chiao Tung University) from The Featured Areas Research Center Program within the framework of the Higher Education Sprout Project by the Ministry of Education (MOE) in Taiwan, and from the Ministry of Education's "Aim for the Top University Plan".}

\section{Author details}

${ }^{1}$ Institute of Biochemistry and Molecular Biology, National Yang Ming Chiao Tung University, Hsinchu 30010, Taiwan. ${ }^{2}$ Cancer Progression Research Center, National Yang Ming Chiao Tung University, Hsinchu 30010, Taiwan. ${ }^{3}$ Institute of Biophotonics, National Yang Ming Chiao Tung University, Hsinchu 30010,

Taiwan. ${ }^{4}$ School of Medicine for International Students, College of Medicine, IShou University, Kaohsiung 82445, Taiwan. ${ }^{5}$ Department of Cell Biology and Anatomy, College of Medicine, National Cheng Kung University, Tainan 70101, Taiwan. ${ }^{6}$ Institute of Physics, Academia Sinica, Taipei 11529, Taiwan

\section{Author contributions}

J.C.K. designed the experiments and wrote the paper. J.W.L, Y.M.H., T.Y.C., T.Y.L., and J.C.K. conducted the experiments and analyzed the data. Y.W.L., H.W.P., and L.R.Y. contributed material and conducted the animal experiments. Y.Q.C. and A.C. contributed material and conducted the image analysis. Y.K.W. and K. H.L. contributed materials and conducted the microcontact printing experiments. All the authors have read and approved the manuscript.

\section{Conflict of interest}

The authors declare no competing interests.

\section{Publisher's note}

Springer Nature remains neutral with regard to jurisdictional claims in published maps and institutional affiliations.

Supplementary information The online version contains supplementary material available at https://doi.org/10.1038/s41420-021-00412-4. 
Received: 20 August 2020 Revised: 27 October 2020 Accepted: 30 October 2020

Published online: 17 February 2021

\section{References}

1. Kislinger, T. et al. Proteome dynamics during C2C12 myoblast differentiation. Mol. Cell. Proteomics 4, 887-901 (2005).

2. Pownall, M. E., Gustafsson, M. K. \& Emerson, C. P. Jr. Myogenic regulatory factors and the specification of muscle progenitors in vertebrate embryos. Annu. Rev. Cell Dev. Biol. 18, 747-783 (2002).

3. Yin, H., Price, F. \& Rudnicki, M. A. Satellite cells and the muscle stem cell niche. Physiol. Rev. 93, 23-67 (2013).

4. Charge, S. B. \& Rudnicki, M. A. Cellular and molecular regulation of muscle regeneration. Physiol. Rev. 84, 209-238 (2004).

5. Allen, D. L., Roy, R. R. \& Edgerton, V. R. Myonuclear domains in muscle adaptation and disease. Muscle Nerve 22, 1350-1360 (1999).

6. Knudsen, K. A. \& Horwitz, A. F. Tandem events in myoblast fusion. Dev. Biol. 58, 328-338 (1977).

7. Wakelam, M. J. The fusion of myoblasts. Biochem. J. 228, 1-12 (1985).

8. Doberstein, S. K., Fetter, R. D., Mehta, A. Y. \& Goodman, C. S. Genetic analysis of myoblast fusion: blown fuse is required for progression beyond the prefusion complex. J. Cell Biol. 136, 1249-1261 (1997).

9. Peckham, M. Engineering a multi-nucleated myotube, the role of the actin cytoskeleton. J. Microsc. 231, 486-493 (2008).

10. Duan, R. \& Gallagher, P. J. Dependence of myoblast fusion on a cortical actin wall and nonmuscle myosin IIA. Dev. Biol. 325, 374-385 (2009).

11. Crawford, G. L. \& Horowits, R. Scaffolds and chaperones in myofibril assembly: putting the striations in striated muscle. Biophys. Rev. 3, 25-32 (2011).

12. Sanger, J. W., Wang, J., Fan, Y., White, J. \& Sanger, J. M. Assembly and dynamics of myofibrils. J. Biomed. Biotechnol. 2010, 858606 (2010).

13. Zhang, T. et al. Microtubule plus-end binding protein EB1 is necessary for muscle cell differentiation, elongation and fusion. J. Cell Sci. 122, 1401-1409 (2009).

14. $\mathrm{Yu}, \mathrm{H}$. et al. Insights into the role of focal adhesion modulation in myogenic differentiation of human mesenchymal stem cells. Stem Cells Dev. 22, 136-147 (2013).

15. Pisaniello, A. et al. The block of ryanodine receptors selectively inhibits fetal myoblast differentiation. J. Cell Sci. 116, 1589-1597 (2003).

16. Burridge, K., Fath, K., Kelly, T., Nuckolls, G. \& Turner, C. Focal adhesions: transmembrane junctions between the extracellular matrix and the cytoskeleton. Annu. Rev. Cell Biol. 4, 487-525 (1988).

17. Hynes, R. O. Integrins: bidirectional, allosteric signaling machines. Cell $\mathbf{1 1 0}$ 673-687 (2002).

18. Jockusch, B. M. et al. The molecular architecture of focal adhesions. Annu. Rev. Cell Dev. Biol. 11, 379-416 (1995).

19. Schwartz, M. A., Schaller, M. D. \& Ginsberg, M. H. Integrins: emerging paradigms of signal transduction. Annu. Rev. Cell Dev. Biol. 11, 549-599 (1995).

20. Zaidel-Bar, R. \& Geiger, B. The switchable integrin adhesome. J. Cell Sci. 123, 1385-1388 (2010).

21. Zaidel-Bar, R., Itzkovitz, S., Ma'ayan, A., lyengar, R. \& Geiger, B. Functional atlas of the integrin adhesome. Nat. Cell Biol. 9, 858-867 (2007).

22. Kuo, J. C., Han, X., Hsiao, C. T., Yates, J. R. 3rd \& Waterman, C. M. Analysis of the myosin-II-responsive focal adhesion proteome reveals a role for beta-Pix in negative regulation of focal adhesion maturation. Nat. Cell Biol. 13, 383-393 (2011).

23. Pletjushkina, O. J. et al. Maturation of cell-substratum focal adhesions induced by depolymerization of microtubules is mediated by increased cortical tension. Cell Adhes. Commun. 5, 121-135 (1998).

24. Riveline, D. et al. Focal contacts as mechanosensors: externally applied local mechanical force induces growth of focal contacts by an mDia1-dependent and ROCK-independent mechanism. J. Cell Biol. 153, 1175-1186 (2001).

25. Chrzanowska-Wodnicka, M. \& Burridge, K. Rho-stimulated contractility drives the formation of stress fibers and focal adhesions. J. Cell Biol. 133, 1403-1415 (1996).

26. Kuo, J. C. Mechanotransduction at focal adhesions: integrating cytoskeletal mechanics in migrating cells. J. Cell. Mol. Med. 17, 704-712 (2013).

27. Gupton, S. L. \& Waterman-Storer, C. M. Spatiotemporal feedback between actomyosin and focal-adhesion systems optimizes rapid cell migration. Cell 125, 1361-1374 (2006).
28. Engler, A. J., Sen, S., Sweeney, H. L. \& Discher, D. E. Matrix elasticity directs stem cell lineage specification. Cell 126, 677-689 (2006).

29. Quach, N. L., Biressi, S., Reichardt, L. F., Keller, C. \& Rando, T. A. Focal adhesion kinase signaling regulates the expression of caveolin 3 and beta1 integrin, genes essential for normal myoblast fusion. Mol. Biol. Cell 20, 3422-3435 (2009).

30. Schwander, M. et al. Beta1 integrins regulate myoblast fusion and sarcomere assembly. Dev. Cell 4, 673-685 (2003).

31. Hollenbeck, P. J. The distribution, abundance and subcellular localization of kinesin. J. Cell Biol. 108, 2335-2342 (1989).

32. Wang, Z. et al. Kif5b controls the localization of myofibril components for their assembly and linkage to the myotendinous junctions. Development 140 , 617-626 (2013).

33. Charvet, B., Ruggiero, F. \& Le Guellec, D. The development of the myotendinous junction. A review. Muscles Ligaments Tendons J. 2, 53-63 (2012).

34. Selva-O'Callaghan, A. et al. Muscle inflammation, autoimmune Addison's disease and sarcoidosis in a patient with dysferlin deficiency. Neuromuscul. Disord. 16, 208-209 (2006)

35. Prelle, A. et al. Clinical, morphological and immunological evaluation of six patients with dysferlin deficiency. Acta Neuropathol. 105, 537-542 (2003).

36. Confalonieri, P. et al. Muscle inflammation and MHC class I up-regulation in muscular dystrophy with lack of dysferlin: an immunopathological study. J. Neuroimmunol. 142, 130-136 (2003).

37. Hoffman, E. P., Rao, D. \& Pachman, L. M. Clarifying the boundaries between the inflammatory and dystrophic myopathies: insights from molecular diagnostics and microarrays. Rheum. Dis. Clin. North Am. 28, 743-757 (2002).

38. Gallardo, E. et al. Inflammation in dysferlin myopathy: immunohistochemical characterization of 13 patients. Neurology 57, 2136-2138 (2001).

39. Klinge, L. et al. New aspects on patients affected by dysferlin deficient muscular dystrophy. J. Neurol. Neurosurg. Psychiatry 81, 946-953 (2010).

40. Fanin, M. \& Angelini, C. Muscle pathology in dysferlin deficiency. Neuropathol. Appl. Neurobiol. 28, 461-470 (2002).

41. Manzur, A. Y., Kuntzer, T., Pike, M. \& Swan, A. Glucocorticoid corticosteroids for Duchenne muscular dystrophy. Cochrane Database Syst. Rev. CD003725, https://doi.org/10.1002/14651858.CD003725.pub3 (2008).

42. Angelini, C. The role of corticosteroids in muscular dystrophy: a critical appraisal. Muscle Nerve 36, 424-435 (2007).

43. Belanto, J. J. et al. Dexamethasone induces dysferlin in myoblasts and enhances their myogenic differentiation. Neuromuscul. Disord. 20, 111-121 (2010).

44. Wagatsuma, A. \& Sakuma, K. Mitochondria as a potential regulator of myogenesis. Sci. World J. 2013, 593267 (2013).

45. Yaffe, M. P. Dynamic mitochondria. Nat. Cell Biol. 1, E149-E150 (1999).

46. Varadi, A. et al. Cytoplasmic dynein regulates the subcellular distribution of mitochondria by controlling the recruitment of the fission factor dynaminrelated protein-1. J. Cell Sci. 117, 4389-4400 (2004).

47. Lihavainen, E., Makela, J., Spelbrink, J. N. \& Ribeiro, A. S. Mytoe: automatic analysis of mitochondrial dynamics. Bioinformatics 28, 1050-1051 (2012).

48. Tanaka, $Y$. et al. Targeted disruption of mouse conventional kinesin heavy chain, kif5B, results in abnormal perinuclear clustering of mitochondria. Cell 93, 1147-1158 (1998)

49. Guo, X. et al. The GTPase dMiro is required for axonal transport of mitochondria to Drosophila synapses. Neuron 47, 379-393 (2005).

50. Hopkins, S. C., Vale, R. D. \& Kuntz, I. D. Inhibitors of kinesin activity from structure-based computer screening. Biochemistry 39, 2805-2814 (2000).

51. Lee, C. M. Transport of C-MYC by Kinesin-1 for proteasomal degradation in the cytoplasm. Biochim. Biophys. Acta 1843, 2027-2036 (2014).

52. Disatnik, M. H. \& Rando, T. A. Integrin-mediated muscle cell spreading. The role of protein kinase $c$ in outside-in and inside-out signaling and evidence of integrin cross-talk. J. Biol. Chem. 274, 32486-32492 (1999).

53. Schlaepfer, D. D., Mitra, S. K. \& llic, D. Control of motile and invasive cell phenotypes by focal adhesion kinase. Biochim. Biophys. Acta 1692, 77-102 (2004).

54. Sloboda, R. D. Isolation of microtubules and microtubule-associated proteins using Paclitaxel. Cold Spring Harb. Protoc. 2015, pdb prot081190 (2015).

55. Hernandez-Alvarez, M. I. et al. Glucocorticoid modulation of mitochondrial function in hepatoma cells requires the mitochondrial fission protein Drp1. Antioxid. Redox Signal. 19, 366-378 (2013).

56. Glater, E. E., Megeath, L. J., Stowers, R. S. \& Schwarz, T. L. Axonal transport of mitochondria requires milton to recruit kinesin heavy chain and is light chain independent. J. Cell Biol. 173, 545-557 (2006). 
57. Rice, S. E. \& Gelfand, V. I. Paradigm lost: milton connects kinesin heavy chain to miro on mitochondria. J. Cell Biol. 173, 459-461 (2006).

58. Gullberg, D., Velling, T., Lohikangas, L. \& Tiger, C. F. Integrins during muscle development and in muscular dystrophies. Front. Biosci. 3, D1039-D1050 (1998).
59. Peet, D. R., Burroughs, N. J. \& Cross, R. A. Kinesin expands and stabilizes the GDP-microtubule lattice. Nat. Nanotechnol. 13, 386-391 (2018).

60. Ruiz, S. A. C. \& Chen, C. S. Microcontact printing: a tool to pattern. Soft Matter 3 11 (2007). 